\title{
Retrieval of ionospheric profiles from the Mars Express MARSIS experiment data and comparison with radio occultation data
}

\author{
B. Sánchez-Cano ${ }^{1,2}$, O. Witasse ${ }^{3}$, M. Herraiz ${ }^{1,2}$, S. M. Radicella ${ }^{4}$, J. Bauer ${ }^{3,5}$, P.-L. Blelly ${ }^{6}$, and G. Rodríguez-Caderot ${ }^{7}$ \\ ${ }^{1}$ Universidad Complutense de Madrid (UCM), Departamento de Física de la Tierra, Astronomía y Astrofísica I, \\ Facultad de Ciencias Físicas, Av. Complutense s/n, 28040 Madrid, Spain \\ ${ }^{2}$ Instituto de Geociencias (UCM-CSIC), c/José Antonio Nováis 2, 28040 Madrid, Spain \\ ${ }^{3}$ European Space Agency, ESTEC - Research and Scientific Support Department, Keplerlaan 1, \\ 2200 AG Noordwijk, The Netherlands \\ ${ }^{4}$ Abdus Salam International Centre for Theoretical Physics (ICTP), Telecommunications/ICT for Development Laboratory, \\ Strada Costiera 11, 34151 Trieste, Italy \\ ${ }^{5}$ European Space Agency, ESOC, Robert-Bosch Strasse 5, 64293 Darmstadt, Germany \\ ${ }^{6}$ Institut de Recherche en Astrophysique et Planétologie 9, Avenue du Colonel Roche, 31028 Toulouse Cedex 4, France \\ ${ }^{7}$ Universidad Complutense de Madrid (UCM), Sección Departamental de Astronomía y Geodesia, Facultad de Ciencias \\ Matemáticas, Av. Complutense s/n, 28040 Madrid, Spain
}

Correspondence to: B. Sánchez-Cano (beasanch@ ifis.ucm.es)

Received: 16 December 2011 - Published in Geosci. Instrum. Method. Data Syst. Discuss.: 25 January 2012

Revised: 2 April 2012 - Accepted: 18 May 2012 - Published: 25 May 2012

\begin{abstract}
Since 2005 the Mars Advanced Radar and Ionospheric Sounding experiment (MARSIS) aboard Mars Express has acquired a unique dataset on the ionosphere of Mars made up of ionospheric soundings taken by the instrument working in its active ionospheric sounding (AIS) mode. These soundings play a role similar to those of modern Terrestrial digisondes in the analysis of our planet ionosphere and have allowed us to dramatically improve our knowledge about the Martian ionosphere. This paper describes this kind of data, which are available from the public Planetary Science Archive, and introduces the MAISDAT tool developed by the European Space Agency to analyze and derive the vertical profile of electron density. Comparisons with radio occultation profiles obtained from Mars Express Radio Science instrument are performed to validate the procedure used in this study.
\end{abstract}

\section{Introduction}

Our current understanding of the ionosphere of Mars has been mostly gained through the radio occultation technique. However, in 2005, Mars Express mission put into operations a low-frequency radar called MARSIS (Mars Advanced
Radar for Subsurface and Ionospheric Sounding) that is designed to sound the subsurface and ionosphere of Mars (Picardi et al., 2004). This technique, in the case of the ionospheric mode, is similar to the method used by digisondes on Earth. Its comparison with previous radio occultation measurements allows us to improve the horizontal spatial resolution and to obtain data at solar zenith angles for which radio occultation cannot be considered (Gurnett et al., 2008). With this instrument, it has been possible to study and understand the behaviour of the Martian ionosphere and its similarities and differences with the Earth ionosphere as never done before. To analyze the Martian ionosphere, MARSIS uses the active ionospheric sounding (AIS) mode and its basic unit of data is the AIS ionogram, which, as will be explained in the next section, is a graph of the delay time (time between the MARSIS radar pulse transmission and its echo reception) versus carrier frequency. Ionograms can be easily converted into vertical electron density profiles.

The knowledge of the ionosphere of Mars has dramatically changed recently thanks to the huge amount of new data. In general terms, the Martian dayside ionosphere is a plasma characterized by two main layers: the M2 layer that corresponds to the maximum ionization area of the ionosphere, and it is produced by the solar EUV photons, and the 
M1 layer that corresponds to the layer below the maximum of ionization and is mainly produced by the soft $\mathrm{X}$-ray photons. In both cases, the ionosphere is mainly composed of $\mathrm{O}_{2}^{+}$. Occasionally, below the M1 layer, a third layer appears sporadically, created by the ablation of meteoroids (Paetzold et al., 2005; Withers, 2009). A significant part of the new information on the Mars ionosphere is due to the harvest that the Mars Express MARSIS radar has produced. One of the most important discoveries by MARSIS instrument has been the finding of the Martian ionopause. Duru et al. (2009) clearly show that the ionopause exists and the average altitude of the boundary, where the magnetic fields change from open to closed, is almost constant and, for solar zenith angles of $60^{\circ}$, is located at approximately $500 \mathrm{~km}$. Some other important discoveries have been made. One of them is the presence of oblique echoes. These kinds of echoes are very common in the MARSIS ionograms, and they are associated with the crustal magnetic field of Mars. It is thought that in these regions there exist bulges in the ionosphere due to the vertical magnetic field. In these areas the sounder detects two different echoes: one due to the vertical reflexion from the ionosphere and the other due to the oblique reflection from the bulge (Gurnett et al., 2005). Another important finding has been the detection of the enhanced electron density over regions where the crustal magnetic field is strong and nearly vertical. This enhanced zone can reach $50 \mathrm{~km}$ above the surrounding ionosphere, and it is believed that the increase in density could be caused by the heating of the electron gas, which leads to a decrease of the recombination coefficient and an increase of the electron density (Duru et al., 2006; Nielsen et al., 2007; Gurnett et al., 2008). Finally, another phenomenon that recently has been revealed is the existence of a new layer at altitudes above about $200 \mathrm{~km}$. Although only one topside layer is usually observed with MARSIS ionograms, sometimes it is possible to detect an additional layer above the maximum ionization. This layer is transitory and lasts about $60 \%$ of the time near the subsolar point. The transient nature of these layers suggests that they originated from a dynamical process, most likely involving an interaction with the solar wind in the upper levels of the ionosphere (Kopf et al., 2008; Gurnett et al., 2008). Furthermore, a "third layer" has been observed in the $1 \%$ of MARSIS AIS mode observations at even higher altitudes (Kopf et al., 2008). Presently, the ionosphere of Mars is much better known and a very large number of MARSIS ionograms is available in the ESA's Planetary Science Archive (PSA).

The main purposes of this study are (1) to describe how the archival data of active ionospheric sounding (AIS) of MARSIS instrument are processed in order to derive the vertical profile of the electron density and (2) to check the correctness of our approach. To achieve the first objective, a description of MARSIS, of the AIS dataset and of the shape and structure of the AIS ionograms is given. Besides, the MAISDAT tool that the European Space Agency has developed to analyze these data and the method to retrieve the ionospheric trace are introduced. In order to meet the second objective, comparisons with radio occultation profiles obtained from Mars Express Radio Science instrument are performed.

\section{Acquisition and processing of the MARSIS AIS data}

The Mars Advanced Radar for Subsurface and Ionospheric Sounding (MARSIS) is a low-frequency radar instrument on board Mars Express mission. This instrument consists of a 40-m tip-to-tip electric dipole antenna, a transmitter, a receiver and a digital data processing system (Picardi et al., 2004). Although its primary objective is to observe the distribution of water in the upper Martian crust, it can also operate in three different secondary modes. In particular, the active ionospheric sounding (AIS) mode is used to analyze the Martian ionosphere. In this mode the sounder of the spacecraft sends through the topside of the ionospheric plasma a vertical radio wave of the frequency $f_{\mathrm{w}}$. The waves are propagated through layers of increasing electron density, while its frequency is greater than the plasma frequency of the surrounding plasma. When it reaches the layer with $f_{\mathrm{w}}=f_{\mathrm{p}}\left(f_{\mathrm{p}}\right.$ is the plasma frequency), the wave is reflected and goes back to the sounder, which measures the delay time between the signal emission and its echo reception. This cycle is repeated step by step for a range of wave frequencies covering the whole spectrum between the local plasma frequency at the altitude of the spacecraft and the maximum plasma frequency of the topside (Bauer, 2008). In particular, in this mode, the instrument transmits a $91.4 \mu$ s short monochromatic radio pulse and then listens for the echoes for $7.3 \mathrm{~ms}$, recording 80 samples of the electric field spectral density at a sampling rate of $91.4 \mu \mathrm{s}$. MARSIS can emit radio frequency signals (RF) in a frequency range between 0.1 and $5.5 \mathrm{MHz}$ at a maximum radiated power of $15 \mathrm{~W}$. The maximum plasma frequency peak in the ionosphere of Mars is located at an altitude around $150 \mathrm{~km}$. To record one complete ionogram, this procedure is repeated for a set of 160 different frequencies, lasting $1.26 \mathrm{~s}$ in total. An ionogram is recorded every $7.54 \mathrm{~s}$. The acquisition of MARSIS data is strongly controlled by the spacecraft orbit. Mars Express goes over a highly eccentric orbit with a periapsis altitude of about $275 \mathrm{~km}$, an apoapsis altitude of about $11000 \mathrm{~km}$ and a period of $6.75 \mathrm{~h}$ (Chicarro et al., 2004). Because of signal-to-noise limitations, ionospheric sounding data are only collected when the spacecraft is near periapsis. A typical MARSIS ionospheric sounding pass starts at an altitude of about $1200 \mathrm{~km}$, continues through periapsis at about $275 \mathrm{~km}$, and ends at an altitude of about $1200 \mathrm{~km}$. The total duration of an ionospheric sounding pass usually is about $40 \mathrm{~min}$ (Gurnett et al., 2005), and around 280 ionograms can be recorded. However, as the instrument has other modes of operation (the main one is the subsurface mode, whose objective is to map the Martian subsurface to study the distribution of water in the upper layer of the Martian crust; Picardi et al., 2005), it is very common to find AIS 
for $10 \mathrm{~min}$, then subsurface mode around the pericenter, and then AIS again.

An AIS ionogram (Fig. 1) is a two-dimensional plot of time delay and frequency. It represents the echo delay time for a series of signals with varying frequency. The frequency varies along the $\mathrm{x}$-axis in the image and the signal delay along the inverted $y$-axis. The echo is visualized by a colour coded spectral density of the E-field component of the returning electromagnetic wave. Starting at zero, the response delay increases with frequency. This part of the response trace is called "ionospheric echo" and corresponds to frequencies reflected in the ionosphere. When the frequency approaches the maximum plasma frequency $f_{\mathrm{p}}(\max )$, the delay increases dramatically due to the decreasing slope of the function $f_{\mathrm{p}}(z)$. At the frequency just above $f_{\mathrm{p}}(\max )$, a cusp indicates when the signal frequency is high enough to pierce the ionospheric layer. This feature comes, because, as the frequency increases together with the electron density, the pulse velocity decreases almost to zero at the critical frequency of the layer. If frequency keeps increasing, the waves will reach the surface of the planet and experiment the reflection on the surface (Bauer, 2008). In the ionograms recorded close to the periapsis, almost always appear vertical lines at different frequencies called "plasma oscillation harmonics". They come from the electrostatic plasma oscillations, which are detected by MARSIS receiver due to specific technical characteristics. These harmonics can actually be used to derive the local plasma frequency by measuring the spacing between the vertical lines (Duru et al., 2008). Other important features that appear sometimes in the AIS ionograms are the horizontal lines located on the left side of the ionograms and equally spaced in delay time. They are known as "electron cyclotron echoes", and their time spacing can be correlated with the cyclotron frequency of the local crustal magnetic field crossed by Mars Express (Gurnett et al., 2008).

The MARSIS AIS dataset is available in the ESA's Planetary Science Archive (PSA). This type of data is written in binary files, where each one contains all received records from the AIS operation phase of one orbit, i.e. they contain every ionogram. The data files are named FRM_AIS_RDR_XXXX.DAT where XXXX is the orbit number. All the geometrical information concerning the sounding events (coordinates of the spacecraft above Mars, altitude, solar angle conditions ...) is contained in one single file, GEO_MARS.TAB, for all data files. There is also a C-program to read the binary file format. The currently available data correspond to the period June 2005-July 2010, in particular between the orbits 1844-8319 (although not in every orbit have AIS data been recorded).

In order to process these data, the European Space Agency has developed a MATLAB software, called MAISDAT (Bauer, 2008). With this software it is possible, among other many options, to perform the inversion of the ionospheric trace in order to obtain the electron density profiles (Fig. 2), which is the most important information that can be retrieved

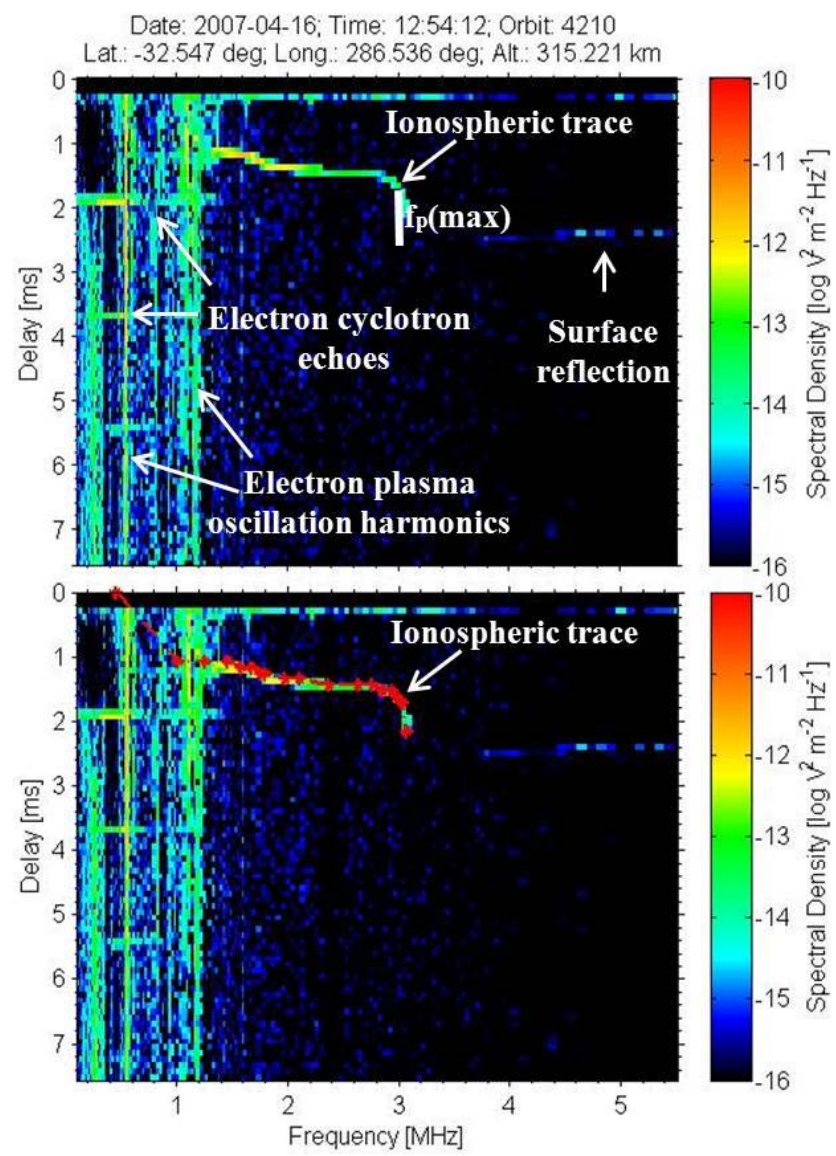

Fig. 1. The top panel shows a typical example of an ionogram obtained from MARSIS instrument using MAISDAT tool. The bottom panel emphasizes the identification of the trace for the ionogram reduction. In red it shows the identification of the trace as it is described in this paper.

from the AIS data. To perform this operation, also known as "ionogram reduction", MAISDAT uses a method based on an inversion of the integral Eq. (1):

$\Delta t\left(f_{\mathrm{w}}\right)=\frac{2}{c} \int_{z_{\text {refl }}}^{z_{\text {sc }}} \frac{\mathrm{d} z}{\sqrt{1-\left(f_{\mathrm{p}}(z) / f_{\mathrm{w}}\right)^{2}}}$

whose formal solution is the Abel's Eq. (2):

$\tilde{z}\left(f_{\mathrm{p}}\right)=\frac{c}{\pi} \int_{\alpha_{0}}^{\pi / 2} \Delta t\left(f_{\mathrm{p}} \sin \alpha\right) \mathrm{d} \alpha$.

$\Delta t$ is the delay time; $f_{\mathrm{w}}$ is the used frequency; $f_{\mathrm{p}}$ is the plasma frequency; $c$ is the light speed; $z_{\mathrm{sc}}$ is the spacecraft altitude; $z_{\text {refl }}$ is the altitude of the ionospheric reflexion; $\sin \alpha=\frac{f_{\mathrm{w}}}{f_{\mathrm{p}}}$ with $f_{\mathrm{w}} \leq f_{\mathrm{p}}$ and $\sin \alpha_{0}=\frac{f_{\mathrm{p}}\left(z_{\mathrm{sc}}\right)}{f_{\mathrm{p}}}$ (Gurnett et al., 2008).

When MAISDAT is initiated, the main window that appears is a spectrogram (a sounding frequency versus time 


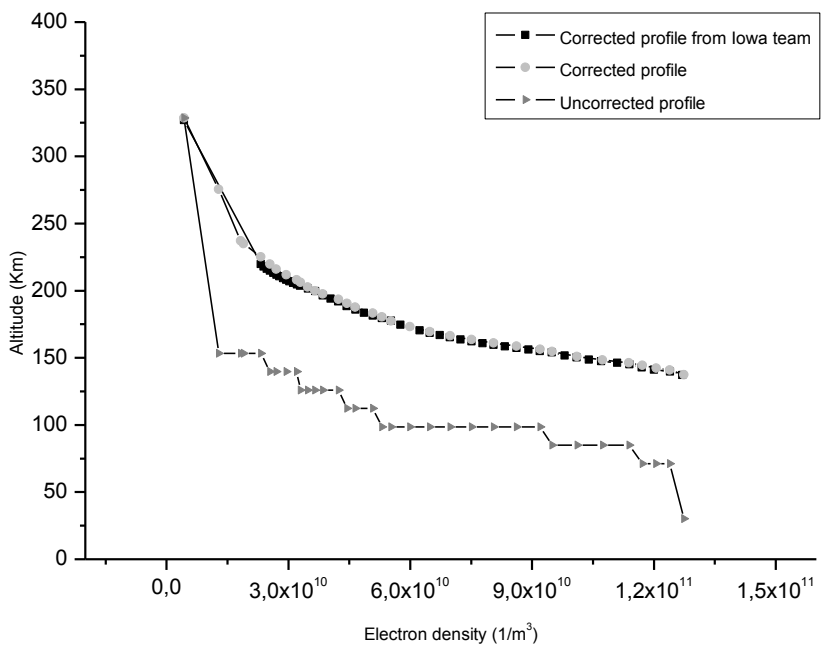

Fig. 2. Example of topside ionospheric profiles obtained from the ionogram 178 in the orbit 2405 of Mars Express. There are two different kinds of profiles: the corrected profile where the true height corrected by the oscillation harmonic in the frequency is used and the uncorrected one where the virtual height is considered. Another corrected profile, obtained at Iowa University by the MARSIS team, is also displayed. The agreement with the ionogram corrected by our team is very remarkable and confirms that the same method is applied to obtain the electron density.

plot), which is composed by stacking all the ionograms contained in the data file along their delay axis. The spectral density of the sounding echo for delay time and frequency values is displayed colour coded in the units: $\mathrm{V}^{2} \mathrm{~m}^{-2} \mathrm{~Hz}^{-1}$. This window offers several options: selecting a data file, opening an ionogram display, opening a time series display or setting the range of spectral density values to be mapped to the colour scale. To analyze the ionospheric trace, it is necessary to start selecting an orbit and an ionogram. Then, a new window with the ionogram tool display described above is shown.

To begin the inversion, it is necessary to know the plasma density in the vicinity of the spacecraft, because the corrected range for each data point depends on the plasma density profile of the sounding wave path (Fig. 1). This information can be obtained from the harmonics of the local plasma frequency, as it is explained in Gurnett et al. (2008). These harmonics (vertical lines) are easily measured from their average frequency separation by digitizing the plot. Then, to determine the ionospheric trace, it is possible to follow either an automatic or a manual procedure. In the first case, it is usually complicated to select the correct trace due to several strong interferences at frequencies below $1 \mathrm{MHz}$. In the second case, this difficulty can be overcome by the user's visual inspection. We propose to select a clear ionogram with a well-defined vertical signature of the trace in the highest frequencies, and to click on the bottom of this part of the trace, because the corresponding frequency is the $f_{\mathrm{p}}(\max )$, which is directly proportional to the maximum ionization in the ionosphere. To select and digitize the rest of the trace until the lowest frequency, where it is no longer distinguishable from noise, we propose to click on the top of the trace, because it is the first record of the refection signal. Then, the digitized information is analyzed by MAISDAT, which gives the corrected and uncorrected electron density profiles of the ionogram. The corrected profiles use the true height in the ionosphere corrected by the oscillation harmonic in the frequency, and the uncorrected profiles use the virtual height. It is important to note that these profiles only explore the top of the ionosphere, i.e. the plasma that exists from the place where Mars Express spacecraft is located to the region of maximum electron density. The electron density measure accuracy is about $\pm 2 \%$, and the uncertainty of the altitude apparent range is about $\pm 6.8 \mathrm{~km}$. (Morgan et al., 2008).

The top of Fig. 2 shows a typical profile from MARSIS radar with the corrected and uncorrected profiles obtained from the 178 ionogram of the orbit 2405. For comparisons, this figure also includes another corrected profile from the same ionogram obtained by the Iowa University team. The results match pretty well.

\section{Comparison with Mars Express radio occultation data}

Mars Express spacecraft carries on board another instrument that is able to take measurements of the Martian ionosphere: the Mars Radio Science (MaRS) instrument. This experiment uses the radio occultation technique to sound the ionosphere and to derive vertical electron density profiles. This procedure is completely different to the sounder method. In general terms, MARSIS sounder sends a sweep of vertical downward radio signals and takes direct measures of the delay time of those frequencies. In its turn, MaRS sends a radio signal at two frequencies (described below) through the upper atmosphere along the spacecraft-Earth line just at the moment when the spacecraft is occulted to the Earth antennas by Mars. From the change in the propagation path and the doppler effect on the signals, it is possible to retrieve the electron density profiles. Due to these differences in the technique, the profiles from MaRS are different from those given by MARSIS. While MaRS allows obtaining the full profile of the ionosphere, MARSIS only allows obtaining profiles from the topside to the maximum ionization peak. In addition, as MaRS requires an occultation, which occurs only during limited periods, and only once per orbit in the case of Mars Express, MARSIS provides a better planet coverage and horizontal spatial resolution and can work in a larger solar zenith angle range.

Radio occultation measurements are remote sensing techniques used for measuring physical properties of a planetary ionosphere. When the spacecraft is going to be occulted to the Earth by Mars, the spacecraft sends radio signals in the $\mathrm{X}$ 


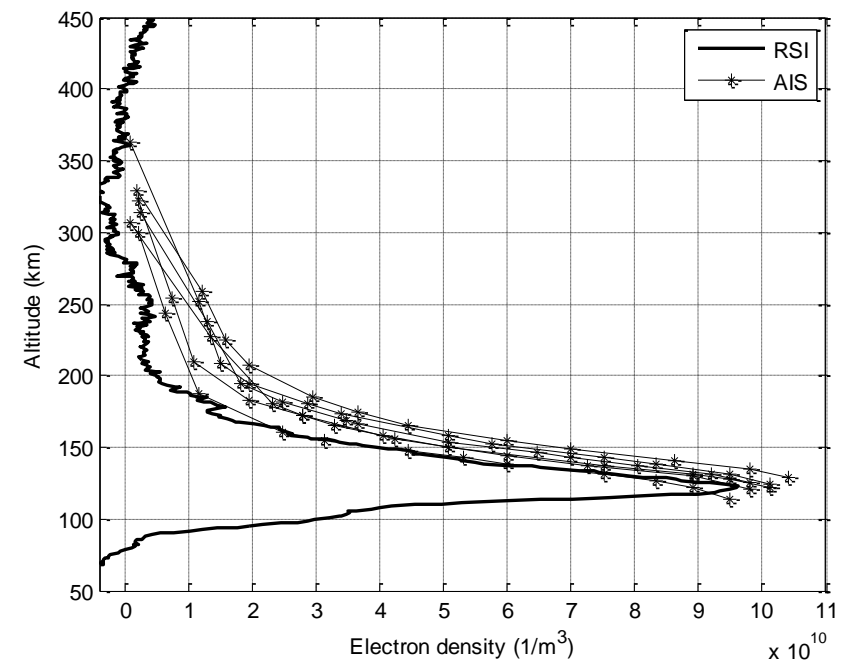

Fig. 3. First Comparison between a profile obtained from Mars Express Radio Science (Year: 2004, DOY: 180, Latitude: $10.2471^{\circ}$, $\mathrm{SZA}=74.6059^{\circ}, \mathrm{Ls}=53.21^{\circ}, F_{10.7}=85$ ) (bold line) and some topside profiles obtained from MARSIS instrument with similar conditions of location, solar zenith angle, solar activity and solar longitude (Orbit: 3065 , ionograms: $20-40$, SZA $=61-65^{\circ}$, Latitude $=11-$ $\left.1^{\circ}, \mathrm{Ls}=59.65^{\circ}, F_{10.7}=80\right)$ (light lines).

and $\mathrm{S}$ bands, which go across Mars's ionosphere. The propagation path of these signals changes as soon as they reach deeper layers in the atmosphere, and the bending angle informs about the electron density distribution in this path of ionosphere. Those ionospheric profiles were retrieved from archival data, using the standard scheme for processing radio occultation data (Paetzold et al., 2005).

Radio science is a well-known technique that has been used widely in the study of the Earth's and other planets' ionospheres. Therefore, in this study we consider these data as a reference to compare and validate the electron density profiles obtained from MARSIS soundings. To this end, different profiles from both kinds of experiments under similar conditions in terms of solar zenith angle, solar longitude, Martian latitude and solar activity ( $F_{10.7}$ index) have been selected. Most of the time, these data show similar results, especially in the region of maximum ionization where the value of the altitude and the electron density of the maximum peak are practically the same. The biggest differences appear close to the topside of the ionosphere (about $180 \mathrm{~km}$ ) where the presence of other physical processes like vertical transport due to diffusion or penetration of an induced magnetic field can play a significant role.

A typical example of this very good agreement is shown in Fig. 3 where the MARSIS profile matches with the radio occultation profile in the altitude range $130-190 \mathrm{~km}$. The remaining MARSIS profiles are similar in electron density but with a small shift in altitude (by about $10 \%$ ). Close to the maximum peak of ionization, the results are practically the

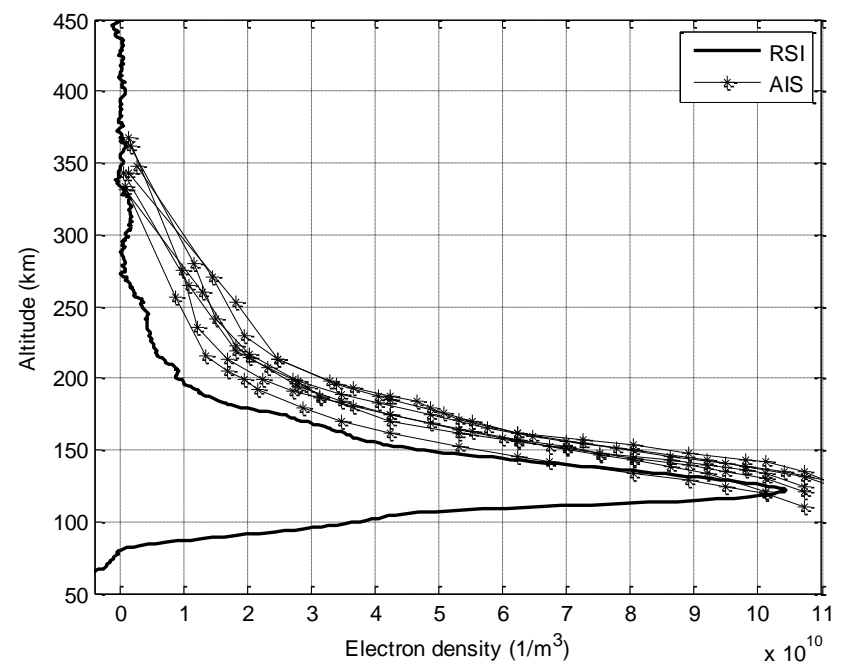

Fig. 4. Second Comparison between a profile obtained from Mars Express Radio Science (Year: 2006, DOY: 084, Latitude: $16.6432^{\circ}$, $\mathrm{SZA}=54.3843^{\circ}, \mathrm{Ls}=30.15^{\circ}, F_{10.7}=76$ ) (bold line) and some topside profiles obtained from MARSIS instrument with similar conditions of location, solar zenith angle, solar activity and solar longitude (Orbit: 5240, ionograms: $155-186, \mathrm{SZA}=50-51^{\circ}$, Latitude $\left.=26-11^{\circ}, \mathrm{Ls}=25.14^{\circ}, F_{10.7}=73\right)($ light lines $)$.

same in both cases with a deviation of about $11 \%$ in altitude and of about $5 \%$ in electron density. With respect to the electron density, the greatest differences reach almost the $50 \%$ of the radio occultation profile at $160 \mathrm{~km}$. As for the altitudes, they can amount to $40 \%$ of the radio occultation profile and appear close to the topside. Figure 4 is another example of this kind of comparison where the results are practically the same.

However, when the difference in some of the considered parameters increases, the degree of similitude in the comparison decreases. Figures 3 and 5 show the same radio occultation profile, which is compared with MARSIS profiles from the same orbit. The only differences between these last profiles are the values of solar zenith angle and latitude, which have increased in the second case. It is possible to observe that in Fig. 5 both kinds of profiles do not match as well as in Fig. 3 where the conditions of every parameter are more similar. This last result indicates that if similar conditions of solar zenith angle, season, latitude and solar activity are not considered, large differences in the adjustments can appear.

This good agreement between the profiles obtained from two different instruments gives us confidence in our data analysis methodology.

\section{Discussion}

In order to describe the ionospheric soundings obtained by MARSIS instrument working in the active ionospheric sounding (AIS) mode, the paper presented the main 


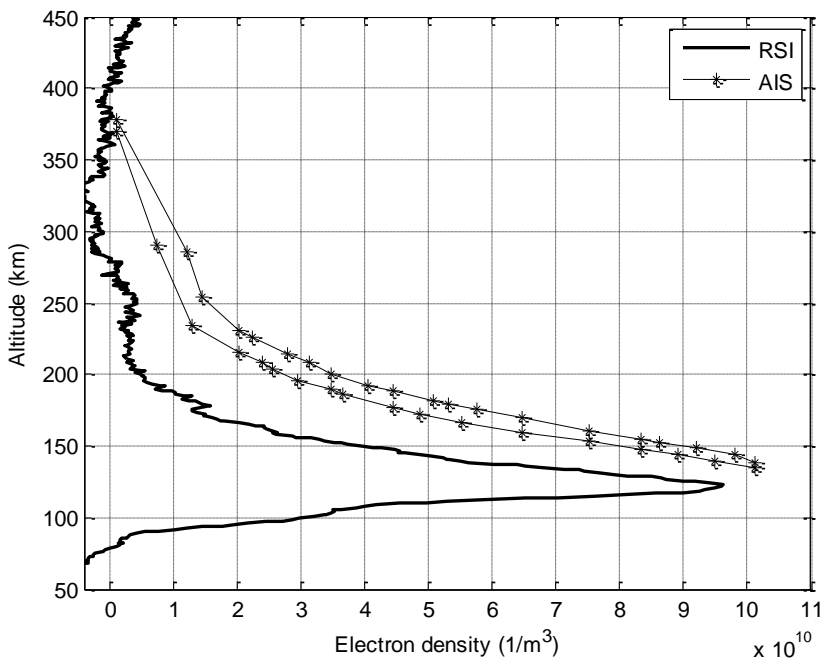

Fig. 5. Third Comparison between a profile obtained from Mars Express Radio Science (The same profile that in Fig. 3, Year: 2004, DOY: 180 , Latitude: $10.2471^{\circ}, \mathrm{SZA}=74.6059^{\circ}, \mathrm{Ls}=53.21^{\circ}$, $F_{10.7}=85$ ) (bold line) and two topside profiles obtained from MARSIS instrument with similar conditions of location, solar activity and solar longitude but different conditions for solar zenith angle (Orbit: 3065 , ionograms: $2-4$, SZA $=57-58^{\circ}$, Latitude $=19.9$ $\left.18.9^{\circ}, \mathrm{Ls}=59.65^{\circ}, F_{10.7}=80\right)$ (light lines).

characteristics of this equipment, the shape and location of the AIS dataset and the structure and particularities of the AIS ionograms. In the same way, the methodology adopted to produce the inversion of these ionograms is described by introducing the MAISDAT tool. This MATLAB utility uses the Abel equation as a base for processing and is able, among other many options, to obtain the electron density distribution.

To start with the inversion routine in MAISDAT following the Bauer (2008) procedure, the first step is to calculate the plasma frequency at the spacecraft altitude using the harmonic lines (vertical lines in the left part of the ionogram). So, it is important to choose ionograms not only with a good ionospheric trace (especially in the highest frequencies), but with good harmonics too. The next step is to select and digitize the ionospheric trace in a manual way, starting with the highest frequencies. In the vertical part of the trace, it is advisable to select the bottom zone and in the rest of the trace the upper part of the pixel, because this part corresponds to the first record of the reflection signal. The manual fitting goes on until the lowest frequency, where the trace is no longer distinguishable from noise. Finally, MAISDAT processes the digitized data to calculate the electron profile of the ionogram.

A comparison between AIS electron density profiles and MaRS radio-science electron density profiles from the Mars Express Radio occultation experiment has been accomplished to validate the ionospheric profiles obtained. As the radio occultation technique is well known in the study of the Earth and planetary ionospheres, it can be considered as a reliable reference. In this study, different profiles from both kinds of experiments with similar conditions of solar zenith angle, solar longitude, Martian latitude and solar activity have been compared. In a large number of cases, the comparison shows similar results, especially in the maximum region of ionization where the values of the altitude and the electron density of the maximum peak are practically the same for both kinds of data. These comparisons give us confidence that the MAISDAT processing is correct.

The Martian ionosphere formation is influenced by many factors. The most typical are the absence of a global magnetic field, the solar radiation and the solar activity. For this reason, one of the most important goals of this current research is to develop a global empirical model for the maximum ionization peak of the dayside ionosphere of Mars. With this purpose the full dataset of MARSIS AIS ionograms is being used. Using MAISDAT software to analyze these data, a sample of ionograms that offers a representative number of profiles in different conditions of latitude, longitude, solar zenith angle, solar activity, solar longitude and Mars-Sun distance was chosen. This set of data has allowed us to develop an empirical model for the main ionospheric peak. A detailed paper on this model will be sent for publication soon.

The $\alpha$-Chapman expression is the basis of this model. This assumes that the mean ionospheric layer is controlled by photo-chemistry only, and the vertical transport is negligible. Ions are created by photo-ionization and ion-neutral reactions, and disappear by radiative or dissociative recombination (Fox, 2009; Sánchez-Cano et al., 2010). In order to obtain and represent mathematically a more realistic behaviour for the ionosphere of Mars, the contribution of different parameters was gradually introduced. In the case of the electron density peak, the first parameter considered in our study was the Sun-Mars distance. This characteristic is very important, because Mars has the most eccentric orbit in the Solar System, and, therefore, the radiation that arrives at Mars depends a lot on the position of Mars with respect to the Sun. With this purpose in mind, and following the Chapman theory, the electron density of every AIS ionogram has been normalized to the electron density that it should have if the ionogram had been taken at the mean orbit distance (1.52 AU). The second parameter considered is the solar zenith angle. This is the most important factor with respect to the electron density of the main peak. The results show a clear exponential trend. The third parameter affecting the electron density peak is the solar activity, in particular, the $F_{10.7}$ index. Other parameters, such as the chemistry and temperature of the Martian upper atmosphere (Forget et al., 2009), the topographic control of the thermal tides in zonal structures (Withers et al., 2003) and the airglow on Mars (Simon et al., 2009), will be considered in a next step of the research. In the case of the altitude peak, as the Chapman theory predicts, the most relevant parameter to be considered is the solar zenith angle. The dependence on this parameter is so important that it may 
mask other parameter effects. Although, the dependence of the height on the solar flux (Mahajan et al., 2009) and on the neutral atmospheric density (Bougher et al., 2001; Zou et al., 2011) is being studied, the solar zenith angle is the only parameter considered for the altitude peak at this stage of the empirical model.

There are also other important parameters that, to a lesser extent, can affect the ionosphere. One of them is the crustal magnetic field in the southern hemisphere of Mars that seems to affect the shape of the biggest and smaller layers in Mars's ionosphere (Nielsen et al., 2007). The effect of this magnetic field, as stated in a previous section, can be clearly observed in the left part of the ionograms, which suggests that the effect of the crustal magnetic field can be important at the spacecraft altitude (Zou et al., 2010).

Acknowledgements. This study is being carried out in the frame work of Project: Participación científica en la misión a Marte MEIGA- METNET PRECURSOR, funded by the Spanish Ministry of Science and Innovation (AYA2009-14212-C05-05/ESP) with a Predoctoral Complutense Grant.

The Mars Express MARSIS and Radio-science data were downloaded from the European Space Agency Planetary Science Archive (http://www.rssd.esa.int/psa). The authors thank A. CardesinMoinelo for his useful support. The MATLAB MAISDAT software and the Bauer (2008) report are available upon request (contact: O. Witasse, European Space Agency, owitasse@ rssd.esa.int).

Edited by: A.-M. Harri

\section{References}

Bauer, J., Analysis of ionospheric sounding data from Mars Express, Master's thesis, Kiruna University, 31 pp., 2008.

Bougher, S. W., Engel, S., Hinson, D. P., and Forbes, J. M.: Mars Global Surveyor Radio Science Electron Density Profiles: Neutral Atmosphere Implications, Geophys. Res. Lett., 28, 30913094, 2001.

Chicarro, A., Martin, P., and Traunter, R.: Mars Express: A European mission to the red planet, European Space Agency Publication Division, SP-1240, Noordwijk, The Netherlands, 3-16, 2004.

Duru, F., Gurnett, D. A., Averkamp, T. F., Kirchner, D. L., Huff, R. L., Persoon, A. M., Plaut, J. J., and Picardi, G.: Magnetically controlled structures in the ionosphere of Mars, J. Geophys. Res., 111, A12204, doi:10.1029/2006JA011975, 2006.

Duru, F.,Gurnett, D. A., Morgan, D. D., Modolo, R., Nagy, A. F., and Najib, D.: Electron densities in the upper ionosphere of Mars from the excitation of electron plasma oscillations, J. Geophy. Res.-Space, 113, A07302, doi:10.1029/2008JA013073, 2008.

Duru, F., Gurnett, D. A., Frahm, R. A., Winningham, J. D., Morgan, D. D., and Howes, G. G.: Steep, transient density gradients in the Martian ionosphere similar to the ionopause at Venus, J. Geophys. Res., 114, A12310, doi:10.1029/2009JA014711, 2009.
Forget, F., Montmessin, F., Bertaux, J.-L., González-Galindo, F., Lebonnois, S., Quémerais, E., Reberac, A., Dimarellis, E., and López-Valverde, M. A.: Density and Temperatures of the Upper Martian Atmosphere Measured by Stellar Occultations with Mars Express SPICAM, J. Geophys. Res., 114, E01004, 114, doi:10.1029/2008JE003086, 2009.

Fox, J. L.: Morphology of the Dayside Ionosphere of Mars: Implications for Ion Outflows, J. Geophys. Res., 114, E12005, doi:10.1029/2009JE003432, 2009.

Gurnett, D. A., Kirchner, D. L., Huff, R. L., Morgan, D. D., Persoon, A. M., Averkamp, T. F., Duru, F., Nielsen, E., Safaeinili, A., Plaut, J. J., and Picardi, G.: Radar soundings of the ionosphere of Mars, Science, 310, 1999-1933, 2005.

Gurnett, D. A., Huff, R. L., Morgan, D. D., Persoon, A. M., Averkamp, T. F., Kirchner, D. L., Duru, F., Akalin, F., Kopf, A. J., Nielsen, E., Safaeinili, A., Plaut, J. J., and Picardi, G.: An overview of radar soundings of the martian ionosphere from the Mars Express spacecraft, Adv. Space Res., 41, 1335-1346, 2008.

Kopf, A. J., Gurnett, D. A., Morgan, D. D., and Kirchner, D. L.: Transient layers in the topside ionosphere of Mars, Geophys. Res. Lett., 35, L17102, doi:10.1029/2008GL034948, 2008.

Mahajan, K. K., Lodhi, N. K., and Singh, S.: Ionospheric effects of solar flares at Mars, Geophys. Res. Lett., 36, L15207, doi:10.1029/2009GL039454, 2009.

Morgan, D. D., Gurnett, D. A., Kirchner, D. L., Fox, J. L., Nielsen, E., and Plaut, J. J.: Variation of the Martian ionospheric electron density from Mars Express radar soundings, J. Geophys. Res., 113, A09303, doi:10.1029/2008JA013313, 2008.

Nielsen, E., Fraenz, M., Zou, H., Wang, J.-S., Gurnett, D. A., Kirchner, D. L., Morgan, D. D., Huff, R., Safaeinili, A., Plaut, J. J., Picardi, G., Winningham, J. D., Frahm, R. A., and Lundin, R.: Local plasma processes and enhanced electron densities in the lower ionosphere in magnetic cusp regions on Mars, Planet. Space Sci., 55, 2164-2172, 2007.

Paetzold, M., Tellmann, S., Häusler, B., Hinson, D., Schaa, R., and Tyler, G. L.: A sporadic third layer in the ionosphere of Mars, Science, 310, 837-839, 2005.

Picardi, G., Biccari, D., Seu, R., Plaut, J., Johnson, W. T. K., Jordan, R. L., Safaeinili, A., Gurnett, D. A., Huff, R., Orosei, R., Bombaci, O., Calabrese, D., and Zampolini, E.: Mars Express: A European mission to the red planet, European Space Agency Publication Division, SP-1240, Noordwijk, The Netherlands, 51-70, 2004.

Picardi, G., Plaut, J. J., Biccari, D., Bombaci, O., Calabrese, D., Cartacci, M., Cicchetti, A., Clifford, S. M., Edenhofer, P., Farrell, W. M., Federico, C., Frigeri, A., Gurnett, D. A., Hagfors, T., Heggy, E., Herique, A., Huff, R. L., Ivanov, A. B., Johnson, W. T. K., Jordan, R. L., Kirchner, D. L., Kofman, W., Leuschen, C. J., Nielsen, E., Orosei, R., Pettinelli, E., Phillips, R. J., Plettemeier, D., Safaeinili, A., Seu, R., Stofan, E. R., Vannaroni, G., Watters, T. R., and Zampolini, E.: Radar soundings of the subsurface of Mars, Science, 310, 1925-1928, 2005.

Sánchez-Cano, B., Herraiz, M., Rodriguez-Caderot, G., and Radicella, S. M.: A study of the ionosphere of Mars: Applications and limitations of the Chapman layer model, Highlights of Spanish Astrophysics VI, Proceedings of the IX Scientific Meeting of the Spanish Astronomical Society, Madrid, Spain, 2010. 
Simon, C., Witasse, O., Leblanc, F., Gronoff, G., and Bertaux, J.-L.: Dayglow on Mars: Kinetic Modelling with SPICAM UV Limb Data, Planet. Space Sci., 57, 1008-1021, 2009.

Withers, P.: A review of observed variability in the dayside ionosphere of Mars, Adv. Space Res., 44, 277-307, 2009.

Withers, P., Bougher, S. W., and Keating, G. M.: The Effects of Topographically-controlled Thermal Tides in the Martian Upper Atmosphere as Seen by the MGS Accelerometer, Icarus, 164, 14-32, doi:16/S0019-1035(03)00135-0, 2003.
Zou, H., Chen, H., Yu, N., Shi, W., Yu, X., Zou, J., and Zhong, W.: Effects of Martian crustal magnetic field on its ionosphere, Sci. China, 53, 1717-1724, 2010.

Zou, H., Lillis, R. J., Wang, J. S., and Nielsen, E.: Determination of seasonal variations in the Martian neutral atmosphere from observations of ionospheric peak height, J. Geophys. Res., 116, E09004, doi:10.1029/2011JE003833, 2011. 